Cercyon quisquilius Linn. and Cryptopleurum minutus Fab.-Two Hydrophyllids living in cowdung, where they are predacious on other insects. More or less cosmopolitan species.

Orytelus sculptus Grav.-A Staphilinid found in cowdung. A cosmopolitan species.

These are the first published records of these beetles in Hawaii. However, Ataenius cognatus is the beetle recorded in the Fauna Hawaiiensis as A. stercorator (Fab.). The six following species of the list have not been previously recorded, but their presence has been known for a number of years, so that they are not to be considered as newly arrived immigrants.

\title{
Report of Committee on Common Names of Economic Insects in Hawaii.
}

In the preparation of its report this Committee has found it advisable to make a few corrections to the list of common names adopted by the Society on March 6, 1913 (published in these Proceedings, vol. 2, pp. 296-300), as well as to present many new names. The correction of the old names has been made necessary through increased knowledge of the habits, distribution, etc., of the insects, or because the old name has proved to be of too general application. Whenever possible a name already in use here or elsewhere has been adopted, especially for the insects found in the lists of common names adopted by the American Association of Economic Entomologists.

P. H. Timberlake,

EDW. M. EHRHORN,

O. H. Swezey,

Committee. 


\section{Coleoptera.}

Algaroba (or Mesquite) weevil.... Bruchus proposis Le Conte. Algaroba (or Mesquite) borer..... Cyllene crinicornis Chevrolat. Australian fern weevil.......... Syagrius fulvitarsis Pascoe. Cadelle (or mealworm).......... Tenebroides mauritanicus (Linnaeus) Chinese rose beetle.............Adoretus sinicus Burmèister.

Dolichos weevil............... Bruchus phaseoli Gyllenhal.

Drug-store beetle............... Sitodrepa panicea (Linnaeus)

Dried-fruit beetle............. Carpophilus hemipterus (Linnaeus)

Fijian Ginger weevil.............Elytroteinus subtruncatus (Fairmaire)

Four-spotted bean weevil......... Bruchus quadrimaculatus (Fabricius)

Glue bush weevil.............. Bruchus sallaei Sharp.

Granary weevil.............. Sitophila granaria Linnaeus.

Gray Acacia ${ }^{\circ}$ weevil............. Bruchus pruininus Horn.

Leather beetle............... Dermestes vulpinus Fabricius.

Little Coconut weevil............ Diocalandra taitense (Guerin)

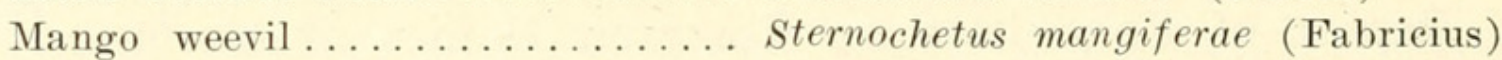

Mexican bean weevil............ Spermophagus pectoralis Sharp.

Mexican bookworm.............. Catorama Mexicana Chevrolat.

Monkey-pod borer............. Xystrocera globosa (Olivier)

Monkey-pod weevil............. Bruchus limbatus Horn.

Rice weevil.............. Sitophila oryzae Linnaeus.

Rusty flour beetle............ Tribolium ferrugineum Fabricius.

Sweet potato weevil............ Cylas elegantulus Summers.

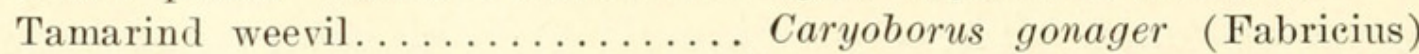

Tamarind snout weevil.......... Sitophila linearis Herbst.

Wardrobe beetle...............Attagenus gloriosae (Fabricius)

West Indian sweet-potato weevil... Euscepes batatae (Waterhouse)

\section{HemipterA.}

Araucaria mealybug...........Eriococcus araucariae Maskell.

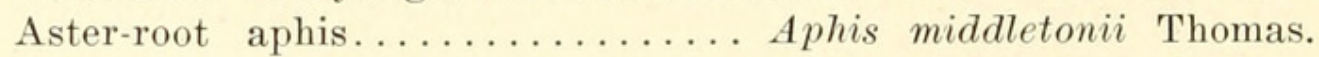

Bur-clover aphis.............. Aphis medicaginis Koch.

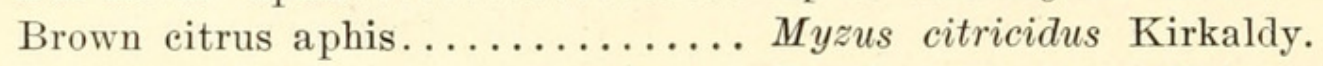

Cinnamon scale..............Eucalymnatus tessellatus (Signoret)

Cotton (or melon) aphis..........Aphis gossypii Glover.

Greenhouse whitefly............Asterochiton vaporariorum(Westwood)

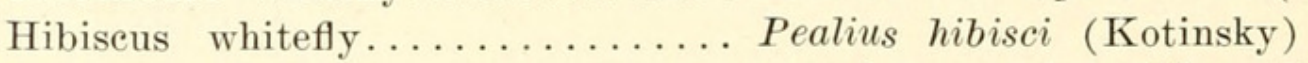

Japanese mealybug.............Pseudococcus kraunhiae (Kuwana)

Palm (or orehid) aphis.......... Cerataphis lataniae (Boisduval)

Soft brown scale............. Coccus hesperidum Linnaeus.

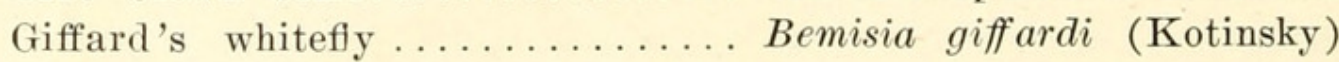




\section{LEPIDOPTERA.}

Cereal moth................. Ephestia elutella Hübner.

Diamond-back moth............ Plutella maculipennis Curtis.

False pink boll-worm........... Pyroderces rileyi (Walsingham)

Hawaiian tobaceo worm.......... Phlegethontius blackburni (Butler)

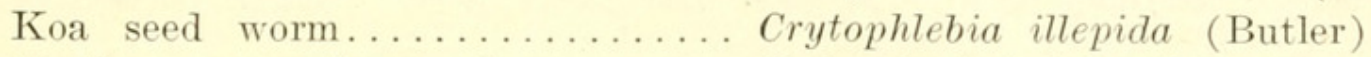

Mediterranean flour-moth......... Ephestia kuhniella Zeller.

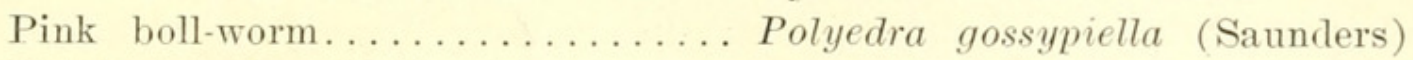

Potato-tuber moth (or tobaceo

split-worm) ........... Phthorimaea operculella (Zeller)

\section{Diptera.}

Cane-borer parasite...................................

Cow-dung sareophaga............ Sarcophaga pallinervis Thomson.

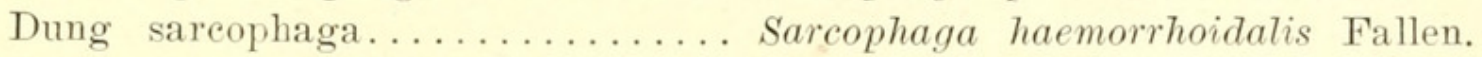

Serpentine leafminer.............Agromyza pusilla Meigen.

\section{Miscellaneous.}

African mole-cricket........... Gryllotalpa africana Fabricius

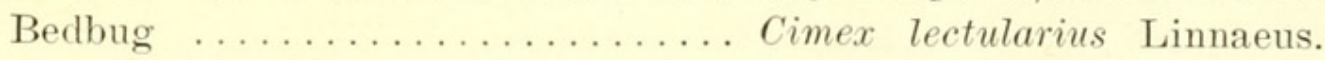

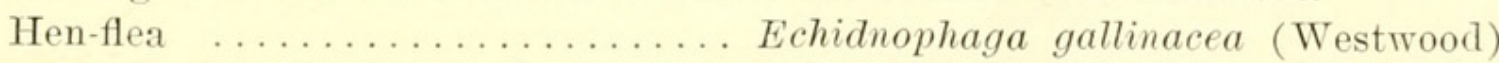

\section{HYMENOPTERA.}

Bruchid egg-parasite............ Uscana semifumipennis Girault.

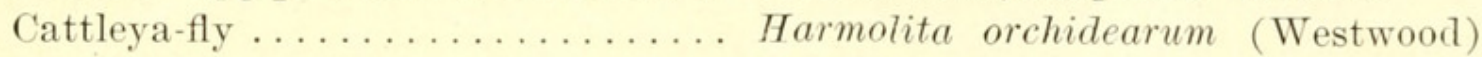

Chinese Dryinid ............... Pseudogonatopus hospes Perkins.

Corn leafhopper parasite......... Paranagrus osborni Fullaway.

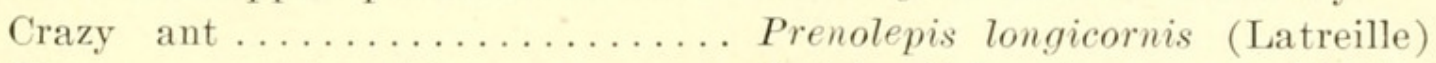

Formosan ootetrastichus.......... Ootetrastichus formosanus Timberlake

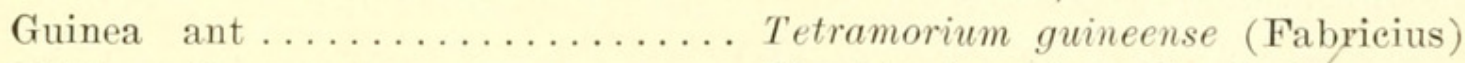

Little yellow ant............ Plagiolepis exigua Forel.

Oriental mealy-bug parasite....... Pauridia peregrina Timberlake.

Sicilian mealy-bug parasite....... Tanaomastix abnormis (Girault.)

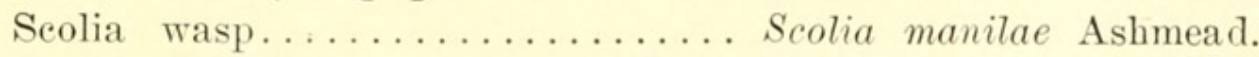




\section{$2 \mathrm{BHL}$ Biodiversity Heritage Library}

Timberlake, P. H., Ehrhorn, E M, and Swezey, Otto H. 1921. "Report of Committee on Common Names of Economic Insects in Hawaii." Proceedings of the Hawaiian Entomological Society 4, 607-609.

https://doi.org/10.5962/bhl.part.16156.

View This Item Online: https://www.biodiversitylibrary.org/item/39842

DOI: https://doi.org/10.5962/bhl.part.16156

Permalink: https://www.biodiversitylibrary.org/partpdf/16156

\section{Holding Institution}

Smithsonian Libraries

\section{Sponsored by}

Smithsonian

\section{Copyright \& Reuse}

Copyright Status: NOT_IN_COPYRIGHT

This document was created from content at the Biodiversity Heritage Library, the world's largest open access digital library for biodiversity literature and archives. Visit BHL at https://www.biodiversitylibrary.org. 